\title{
The Kepler program for observing interesting CVs
}

\author{
Zhibin Dai $^{* a \dagger}$, Paula Szkody $^{b}$, Peter M. Garnavich $^{c}$ and Mark Kennedy ${ }^{c d}$ \\ ${ }^{a}$ Yunnan Observatories, Chinese Academy of Sciences. \\ P. O. Box 110, 650011 Kunming, P. R. China. \\ ${ }^{b}$ University of Washington, Seattle, WA, 98195, USA. \\ ${ }^{c}$ University of Notre Dame, Notre Dame, IN, 46556, USA. \\ ${ }^{d}$ Department of Physics, University College Cork, Cork, Ireland. \\ E-mail: zhibin_daidynao.ac.cn
}

Kepler K2 space telescope observations of cataclysmic variables (CVs) were used to search for periodic behavior and identify possible pseudo periods due to the satellite artefacts from periodic thrusters. Detailed period analyses were carried out for the dwarf nova UV Gem observed in the long cadence (LC) of the K2 campaign 0 (K2-C0) and seven CVs, comprised of four faint CVs and three known dwarf novae QZ Vir, RZ Leo and TW Vir observed in the long and short cadences (LC and SC) of the K2 campaign 1 (K2-C1), respectively. All 23 signals including harmonics and beat periods were identified from the periodograms of the four faint CVs in K2$\mathrm{C} 1$. Among these 23 periods, there were eight signals distinctly detected from the periodogram of J1125-0016 due to a possible resonance between its orbital period and the common 5.9-hr satellite thruster period. A series of periodic dips with varying profiles obeying a period of 3.9256 day were found in all three SC light curves of $\mathrm{K} 2-\mathrm{C} 1$, which were extracted from the original $\mathrm{K} 2$ data files. This period is due to a periodic short-timescale resetting of the thruster. A two-dimensional power spectrum of the dwarf nova UV Gem observed in K2-C0 with a moving window of 4.7 day was derived for demonstrating the variations of the two significant modulations at the periods of $2.07 \mathrm{hr}$ and $2.12 \mathrm{hr}$ during the outburst and quiescent phases. In addition, the other two newly detected weak but consistent signals at the periods of $1.06 \mathrm{hr}$ and $0.91 \mathrm{hr}$ were seen, likely due to the first harmonic of the $2.12 \mathrm{hr}$ period and the fifth harmonic of the 6-hr thruster event of the satellite, respectively.

The Golden Age of Cataclysmic Variables and Related Objects - III, Golden2015

7-12 September 2015

Palermo, Italy

\footnotetext{
${ }^{*}$ Speaker.

${ }^{\dagger}$ Key Laboratory for the Structure and Evolution of Celestial Objects, Chinese Academy of Sciences, P.R.China.
} 


\section{Introduction}

Cataclysmic Variables (CVs) are semidetached binary systems with short orbital periods $\sim 80 \mathrm{~min}$ $10 \mathrm{hr}$, in which a Roche lobe filling red dwarf transfers mass to a white dwarf (WD) via the L1 point. Comprehensive reviews of CVs are given in Warner $(1995,2003)$ [1, 2]. Since the primary Kepler mission can provide nearly continuous light curves for a long time [3, 4], it offers an unprecedented chance for the study of accreting stars and interacting binaries. Consequently, 8 of the $15 \mathrm{CVs}$ successfully monitored by the Kepler space telescope were analyzed in detail [5, 6, 7]. Although the primary Kepler mission was terminated in 2013 May due to the loss of two of the spacecraft's reaction wheels, the entirely community-driven followup mission (K2) continues to provide similar high-precision photometric data thanks to setting the space telescope on the ecliptic plane and pointing at a series of fixed sky areas for a continuous observation period (named campaign) approximately 80 days. Although the first engineering test campaign (K2-C0) only lasted for a short observation period of 35 days, the following formal campaigns have the full-length observation period (i.e., 80 day). Until now, the $\mathrm{K} 2$ mission has carried out 7 campaigns from K2-C0 to K2-C6 since March 2014, and K2-C7 is running. At present, the K2 mission plans to continue operating until 2017 or 2018. Like the primary Kepler mission, the formal campaigns of the K2 mission have two observation modes, i.e., long and short cadences (LC and SC) with 30-min and 1-min sampling rates, respectively. Hence, the long-baseline, high-precision photometry provided by the K2 mission holds an enormous potential for detecting the short-timescale variations in CVs $[3,4,8,9,10]$.

Based on our proposals of the first two campaigns, there were $15 \mathrm{CVs}$ successfully observed, 6 in K2-C0 and 9 in K2-C1. Dai et al. (2016) discussed 8 relatively brighter objects of these 15 CVs [11]. This paper focuses on the detailed period analyses of a well-known dwarf nova UV Gem in $\mathrm{K} 2-\mathrm{C} 0$ and seven $\mathrm{CV}$ s in $\mathrm{K} 2-\mathrm{C} 1$ including the search for possible periods caused by the pointing of the Kepler space telescope during the observing campaigns. Note that 4 of the $7 \mathrm{CVs}$ in $\mathrm{K} 2-\mathrm{C} 1$ were never discussed before since they are relatively faint targets at the detection limit of the satellite.

\section{K2 Light Curve Extractions}

Table 1 lists the 4 faint CVs observed in K2-C1. Considering that the "self-flat-fielding" (SFF) method proposed by Vanderburg \& Johnson (2014) [12] and Vanderburg (2014) [13] may not work well for most CVs light curves [11], the light curves of these 4 were extracted from the original Target Pixel Files (TPFs) by using the program PyKE developed by the Guest Observer Office [14]. They were then transformed to the K2 magnitude by using the transformation formula in Dai et al. (2016) [11]. Vanderburg \& Johnson (2014) [12] and Vanderburg (2014) [13] defined a parameter of improvement percentage to describe the improvement in photometric precision of K2 light curves by using the SFF method. Although 3 of the 4 CVs listed in Table 1 have SFF improvement percentages lower than 50\% and even J1138+0619 has a negative percentage $-0.7 \%$, our deduced K2 light curve of the remaining object 2dF J112555-001639 (hereafter J1125-0016) is compromised due to the serious contamination from the extremely close background object named 2MASS J11255558-0016301 ( $\mathrm{B}=16.75 \mathrm{mag}$ in SIMBAD), which is another K2-C1 target with the Ecliptic Plane Input Catalog (EPIC) identification number 201446083. The position difference 
Table 1: The 4 faint Cataclysmic Variables observed in K2-C1.

\begin{tabular}{|c|c|c|c|c|c|c|}
\hline CV name (EPIC) & $\begin{array}{l}\text { R.A. \& Dec. } \\
\text { J2000 }\end{array}$ & $\begin{array}{l}\text { SFF } \\
\%\end{array}$ & $\begin{array}{c}\mathrm{P}_{\text {orb }} \\
\mathrm{hr}\end{array}$ & $\begin{array}{l}\text { Magnitude } \\
\mathrm{V} / \mathrm{g}\end{array}$ & Type & Ref. \\
\hline${ }^{a} \mathrm{~J} 1125-0$ & $11: 25: 55.72-00: 16: 38.5$ & +325.8 & 1.47 & $19.7 \mathrm{~B}$ & DN & [15] \\
\hline MLS130313 (210282488) & $11: 15: 37.1+05: 10: 01$ & +22 & - & 16.7 & $\mathrm{CV}$ & [16] \\
\hline MLS121203 (21028248 & $11: 37: 51.0+00: 42: 17$ & +1.2 & - & 19. & $\mathrm{CV}$ & [16] \\
\hline${ }^{b} \mathrm{~J} 1138+0619$ (201850883) & $11: 38: 26.73+06: 19: 19.5$ & -0.7 & 1.4 & 18.9 & $\mathrm{CV}$ & [17] \\
\hline
\end{tabular}

Note, ${ }^{a}$ listed in the RKcat7.21 catalogue. ${ }^{b}$ only listed in the CVs catalogue from the SDSS.

between the two targets is only 1.64 arcsec, which is obviously beyond the spatial resolution of the Kepler space telescope ( 3.98 arcsec per pixel). Therefore, they are hard to distinctly separate in the Kepler field. In spite of this, we attempted to use the kepmask tool to define the individually optimal apertures for these two objects. Both light curves extracted from their individually optimal pixel masks were dominated by the unacceptable scatter. Moreover, the light curve extracted from the largest aperture covering two objects became totally flat $(<0.01 \mathrm{mag}$ on average), since the photometric variations were badly smoothed by the brighter background star, which may be a constant star. Thus, the light curve of J1125-0016 shown in the top panel of Figure 1 is directly plotted from the SFF corrected data with the considerably higher improvement percentage $325.8 \%$. The other three K2 light curves in K2 magnitude are shown in the bottom three panels of Figure 1.

\section{Period Analysis}

\subsection{Pseudo Periods in the LC data}

Hakala et al. (2015) and Kraus et al. (2015) pointed out a common period $\sim 6 \mathrm{hr}$ caused by a spacecraft thrusting event $[18,19]$. We expected to determine whether the other possible periods including beat periods can be found in the K2 data. The four CV light curves shown in Figure 1 do not show any outburst or periodic event except for some irregular and occasional flickerings. The periodograms are shown in Figure 2. Among these four CVs, J1125-0016 is a "post period-bounce" dwarf nova with an orbital period of $88 \mathrm{~min}$ [20], and therefore is a special target, which only shows the well known 6-hr satellite period and its harmonics in its periodogram. This feature in the power spectrum was not seen in the other three CVs. Hence, it may be reasonable to consider a resonance phenomenon coincidently occurring around its orbital period, which is nearly equal to one fourth of the thruster period $5.9 \mathrm{hr}$. The resonance can considerably amplify the harmonics relevant to the resonant period $1.47 \mathrm{hr}$. If the assumption of the resonance around the period $1.47 \mathrm{hr}$ is true, then the other signals may have the relative lower powers compared with the amplified power at the period of $1.47 \mathrm{hr}$. Note that both periods are not exactly equal but very close since all the narrow peaks shown in the top panel of Figure 2 are actually doubled, which can be clearly seen in the three inserts of Figure 2, and the largest difference at the thruster period of $5.9 \mathrm{hr}$ with the most significant power is only $1.6 \mathrm{~min}$. The two peaks of the doubled lines in the spectrum at the long 


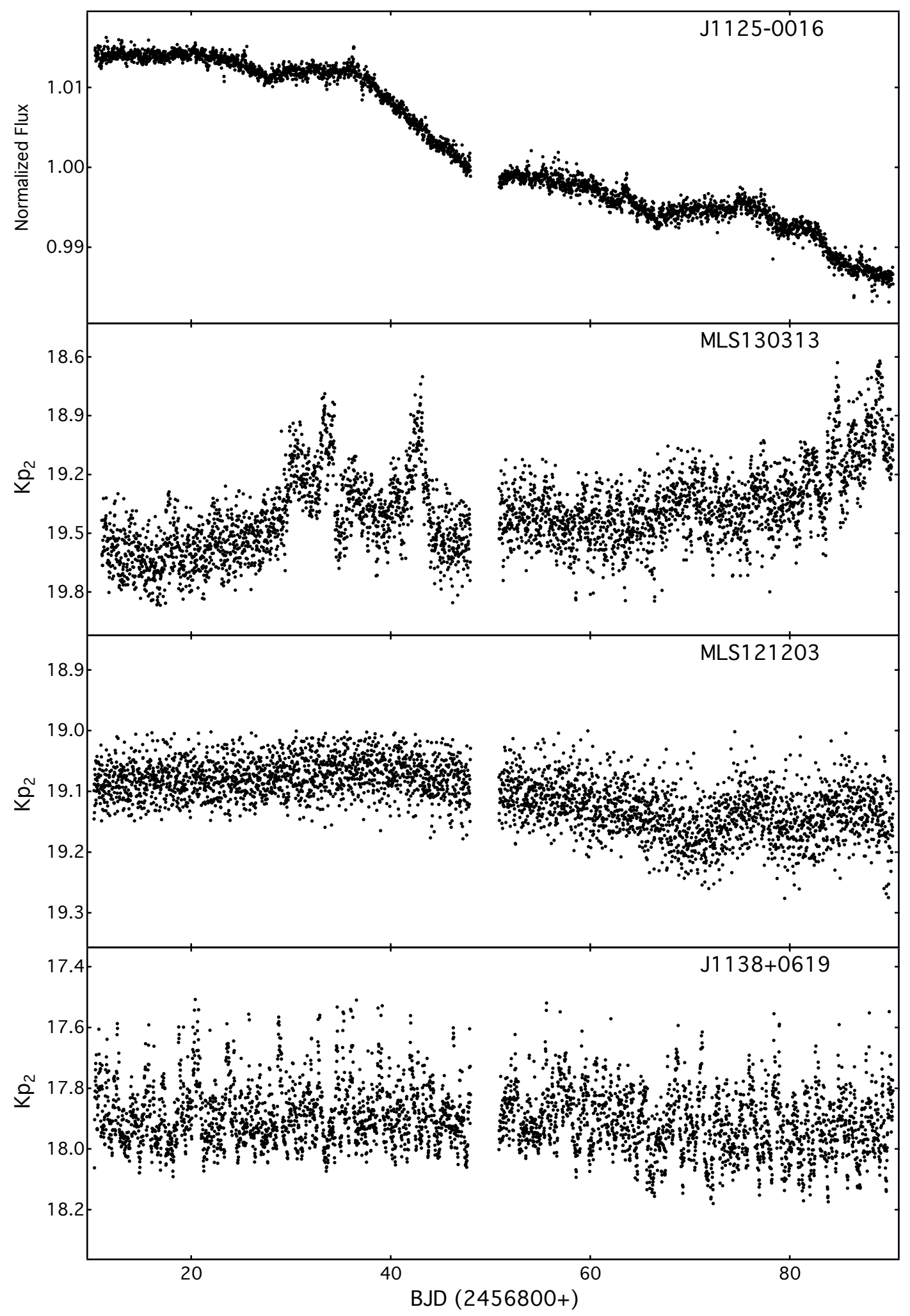

Figure 1: The four faint CVs observed in $\mathrm{K} 2-\mathrm{C} 1$. The top panel is the SFF corrected light curve in normalized SAP flux. But, the other three panels are our deduced K2 light curves in K2 magnitude. 
and short periods are the harmonics and/or beats of the thruster and orbital periods, respectively. Furthermore, the eight signals can be accurately identified from the periodogram of J1125-0016 thanks to the resonance.

The other three panels mainly illustrate the primary harmonic of the 6-hr thruster period including a few high-order harmonics and beats. The peak at period $5.9 \mathrm{hr}$ is the only significant signal in the periodogram of MLS130313. Although the periodogram of MLS121203 never shows any significant signal, we attempted to pick out some signals with relatively high powers for comparisons with the results from the other three targets. The bottom panel of Figure 2 only shows a group of significant peaks around the central period of $\sim 10.3 \mathrm{hr}$. In addition, there is not any peak near to its orbital period of $1.4 \mathrm{hr}$ derived by Szkody et al. (2006) [17]. Table 2 lists all 23 periods with the signal-to-noise larger than 5 marked in Figure 2.

\subsection{Pseudo Periods in the SC data}

During the reduction of the $\mathrm{SC}$ data in $\mathrm{K} 2-\mathrm{C} 1$, we found that their original light curves directly plotted from the output data files of the PyKE display a series of dips, which are shown in Figure 3. However, they were not shown in the plot files output from the PyKE package kepdraw. Considering that these dips exist in the ASCII data files, it is necessary to find out their origins for the followup data analysis. Figure 4 plots the total 38 zoomed dips including 11 in outburst for the K2 light curve of TW Vir. The dips shown in Figure 3 and 4 apparently occur at a period of $\sim 2$ days. But, the L-S technique may not be the best method to search for very sharp features like these dips. Thus, an average separation 1.9628 day between two adjoining dips and the first minimum time of the dips are set as the period and epoch, respectively. Although the O-C analysis is failed by applying this period, a nearly strict period of 3.9256 days can be obtained based on a double of this period (i.e., 3.9256 days). The derived O-Cs shown in Figure 5 clearly indicate a half-cycle (i.e., 3.9256 day per cycle) jump coincidently occurring around the 3-day data gap of K2-C1. Before and after this jump, this period is very stable. The Kepler team attributed this $\sim 4$-day signal to be a periodic thruster realignment with a period of $\sim 48 \mathrm{hr}$, which is the other pseudo period. Furthermore, the half-cycle jump shown in the $\mathrm{O}-\mathrm{C}$ diagram implies that this resetting motion may have two modes. After manually removing these dips, the regular gaps left in the data series may cause aliasing in the period analysis. Therefore, it should be careful to declare the periods detected from the K2-SC data.

\subsection{Modulations in UV Gem}

A smooth K2 light curve of UV Gem with a complete normal outburst was shown in Dai et al. (2016) [11]. They carried out a rough period analysis and found two significant periods of $2.07 \mathrm{hr}$ and $2.12 \mathrm{hr}$. The long period $2.12 \mathrm{hr}$ with less power was regarded as a remaining superhump period after an unrecorded superoutburst. However, both periods are less than the orbital and superhump periods listed in the updated CVs catalog (RKcat 7.21) [15], i.e., $2.15 \mathrm{hr}$ and $2.24 \mathrm{hr}$, respectively, which were similar to the previous results derived by Kato \& Makoto (2001) [21] and Kato et al. (2013) [22]. Since the previously derived orbital period was estimated from the superhump period by using an empirical relation given by Stolz \& Schoembs (1984) [23], we expected to further see these two modulations during the 35-day observations of K2-C0. 


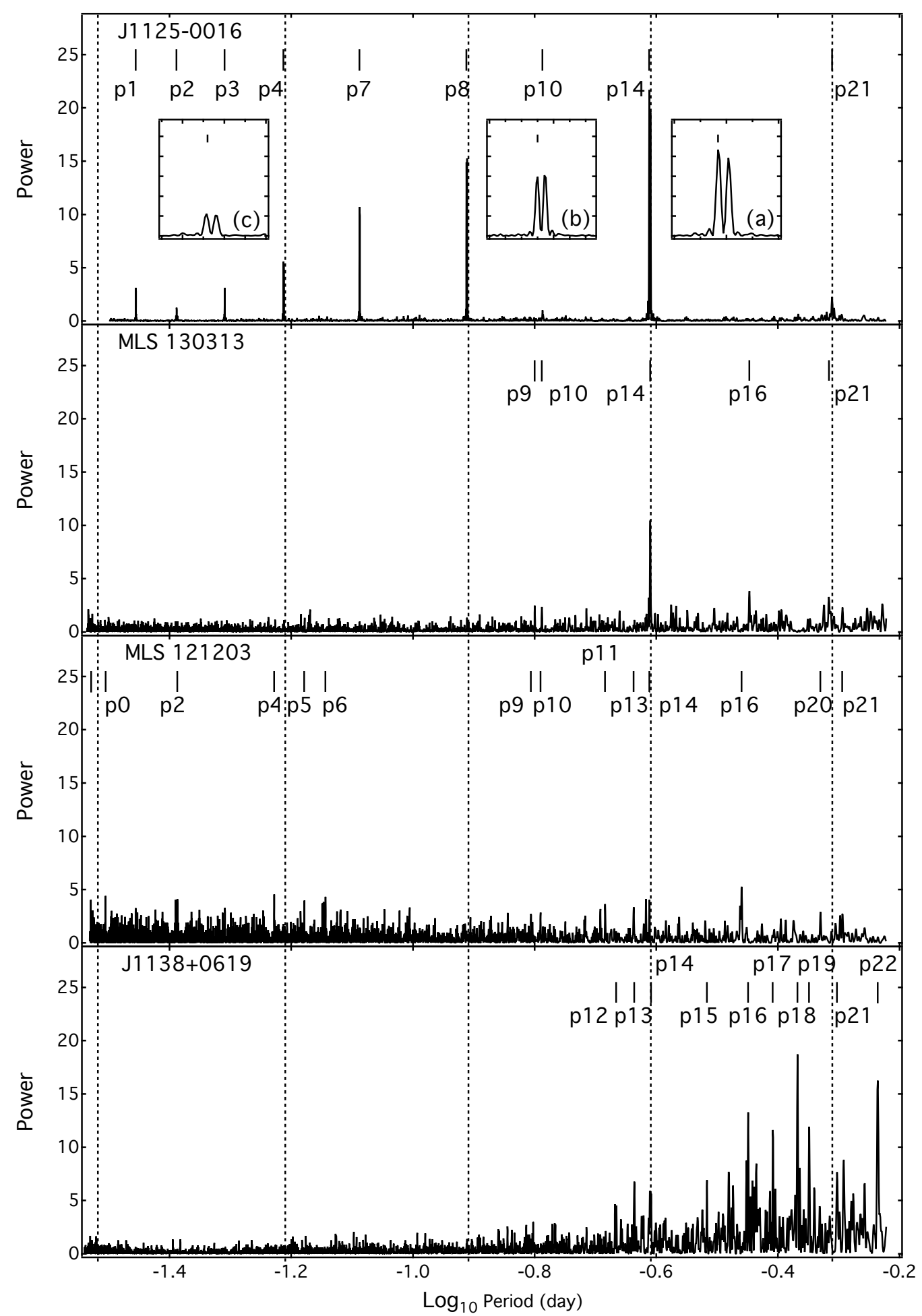

Figure 2: The power spectra of the four K2 light curves plotted in Figure 1 derived from the Lomb-Scargle periodogram method. The periods and their harmonics or beats with the signal-to-noise larger than 5 are marked by the short vertical lines. And the corresponding period IDs are labelled. The five vertical dash lines refer to the 6-hr thruster period and its harmonics. From left to right, they are the $7^{t h}, 3^{r d}, 1^{s t}$ harmonics, thruster period and sub-harmonic, respectively. Note that the periodograms are plotted in common logarithm rather than natural logarithm for visuality. 


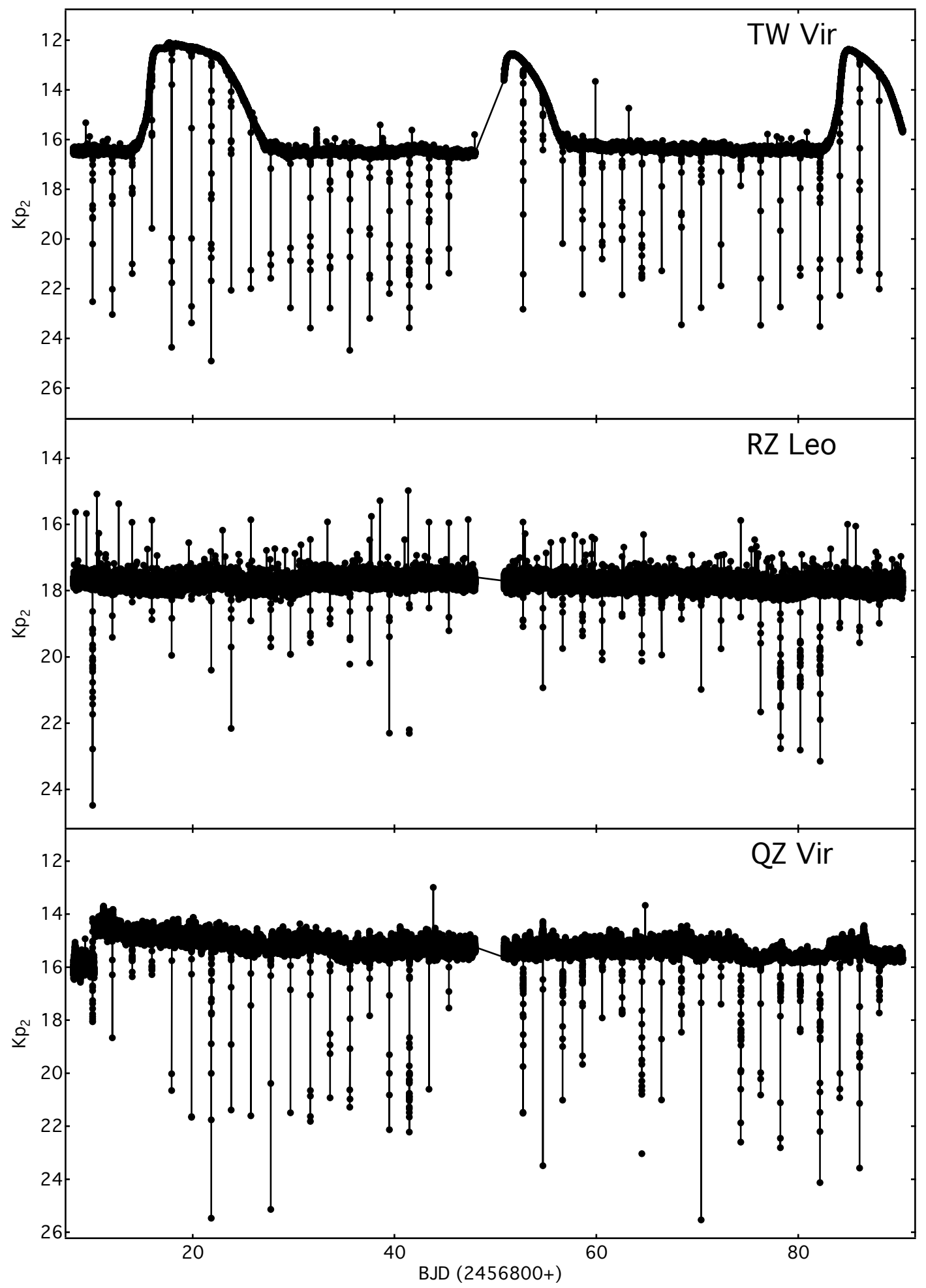

Figure 3: Three SC light curves of the dwarf novae observed in K2-C1. The downward spikes are the dips resulted from the satellite every $\sim 2$ days, while the upward spikes may be the cosmic rays. 

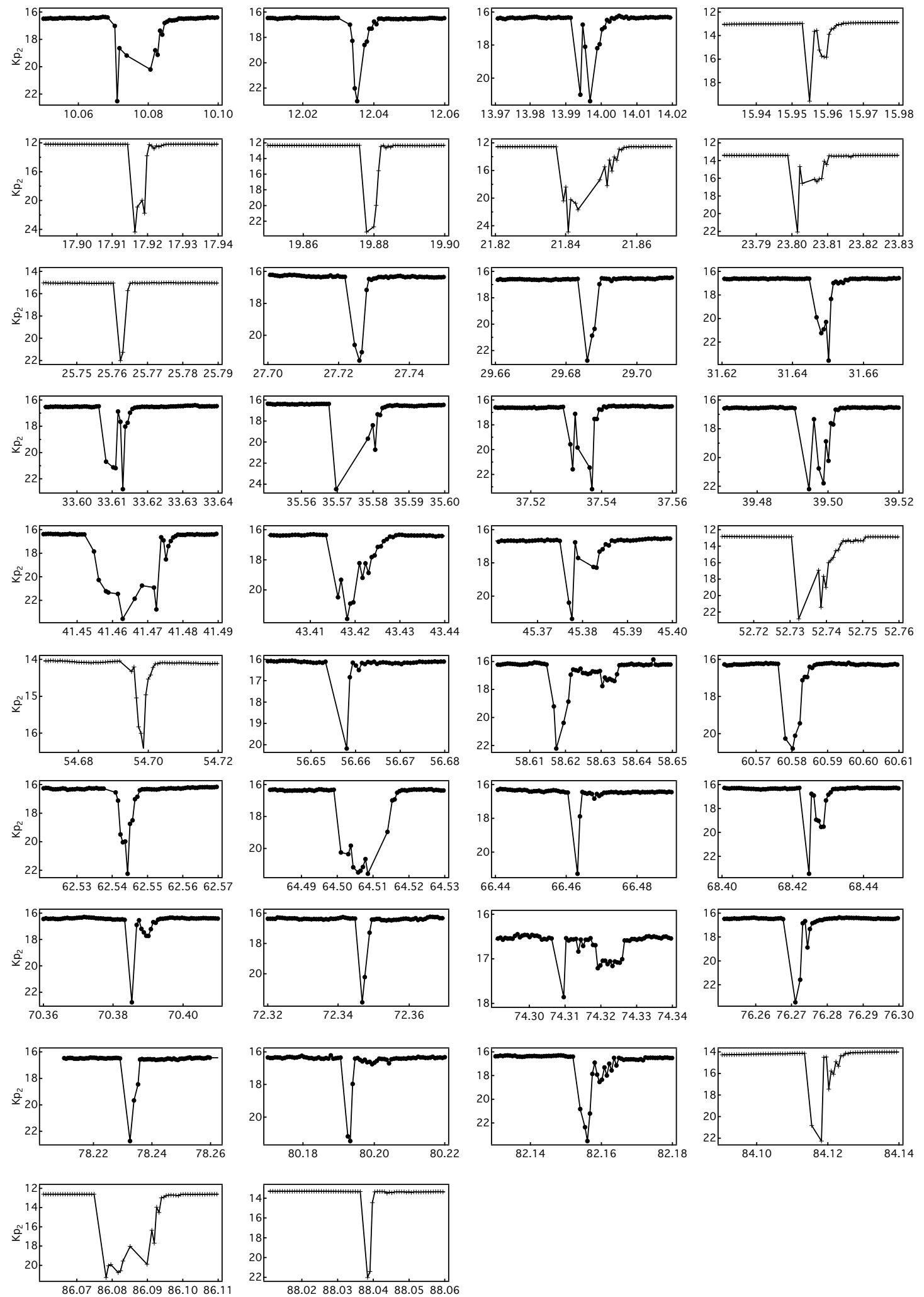

Figure 4: The total 38 zoomed dips (i.e., downward spikes plotted in Figure 3) in the SC light curves of TW Vir. The solid cycles and crosses denote the dips occurring in quiescence and outburst, respectively. 
Table 2: List of periods detected from the periodograms of the 4 faint $\mathrm{CV}$ s observed in $\mathrm{K} 2-\mathrm{C} 1 .^{a}$

\begin{tabular}{|c|c|c|c|c|c|}
\hline \multirow[t]{2}{*}{$\mathrm{ID}^{b}$} & \multicolumn{2}{|c|}{$\mathrm{J} 1125-0016$} & \multirow[t]{2}{*}{ MLS130313 } & \multirow[t]{2}{*}{ MLS121203 } & \multirow[t]{2}{*}{$\mathrm{J} 1138+0619$} \\
\hline & thruster & orbital & & & \\
\hline $\mathrm{p} 0=7^{t h} \mathrm{p} 14$ & \multicolumn{2}{|c|}{-} & - & $0.71 \& 0.75$ & \\
\hline $\mathrm{p} 1=6^{\text {th }} \mathrm{p} 14$ & 0.841 & 0.840 & - & - & - \\
\hline $\mathrm{p} 2=5^{t h} \mathrm{p} 14$ & \multicolumn{2}{|c|}{0.981} & - & 0.984 & - \\
\hline $\mathrm{p} 3=4^{\text {th }} \mathrm{p} 14$ & 1.177 & 1.176 & - & - & - \\
\hline $\mathrm{p} 4=3^{r d} \mathrm{p} 14$ & 1.472 & 1.470 & - & 1.419 & - \\
\hline $\mathrm{p} 5(\mathrm{p} 3, \mathrm{p} 11)^{c}$ & \multicolumn{2}{|c|}{-} & - & 1.591 & - \\
\hline $\mathrm{p} 6(\mathrm{p} 4, \mathrm{p} 18)^{c}$ & \multicolumn{2}{|c|}{ - } & - & 1.723 & - \\
\hline $\mathrm{p} 7=2^{n d} \mathrm{p} 14$ & 1.963 & 1.960 & - & - & - \\
\hline $\mathrm{p} 8=1^{s t} \mathrm{p} 14$ & 2.945 & 2.939 & - & - & - \\
\hline $\mathrm{p} 9(\mathrm{p} 8, \mathrm{p} 22)^{c}$ & \multicolumn{2}{|c|}{-} & 3.804 & 3.752 & - \\
\hline $\mathrm{p} 10(\mathrm{p} 8, \mathrm{p} 21)^{c}$ & \multicolumn{2}{|c|}{3.919} & 3.908 & 3.889 & - \\
\hline $\mathrm{p} 11(\mathrm{p} 0, \mathrm{p} 1)^{c}$ & \multicolumn{2}{|c|}{-} & - & 4.967 & - \\
\hline $\mathrm{p} 12=1^{s t} \mathrm{p} 19$ & \multicolumn{2}{|c|}{-} & - & - & 5.181 \\
\hline $\mathrm{p} 13(\mathrm{p} 9, \mathrm{p} 21)^{c}$ & \multicolumn{2}{|c|}{-} & - & 5.532 & 5.547 \\
\hline $\mathrm{p} 14^{d}$ & 5.896 & 5.869 & 5.892 & 5.874 & 5.910 \\
\hline $\mathrm{p} 15(\mathrm{p} 10, \mathrm{p} 16)^{c}$ & \multicolumn{2}{|c|}{-} & - & - & 7.303 \\
\hline $\mathrm{p} 16(\mathrm{p} 11, \mathrm{p} 21)^{c}$ & \multicolumn{2}{|c|}{-} & 8.573 & 8.330 & 8.537 \\
\hline $\mathrm{p} 17(\mathrm{p} 12, \mathrm{p} 20)^{c}$ & \multicolumn{2}{|c|}{-} & - & - & 9.370 \\
\hline $\mathrm{p} 18(\mathrm{p} 13, \mathrm{p} 21)^{c}$ & \multicolumn{2}{|c|}{-} & - & - & 10.285 \\
\hline $\mathrm{p} 19(\mathrm{p} 13, \mathrm{p} 20)^{c}$ & \multicolumn{2}{|c|}{ - } & - & - & 10.745 \\
\hline $\mathrm{p} 20(\mathrm{p} 13, \mathrm{p} 19)^{c}$ & \multicolumn{2}{|c|}{-} & - & 11.225 & - \\
\hline $\mathrm{p} 21^{e}$ & \multicolumn{2}{|c|}{11.721} & 11.587 & 12.185 & 11.949 \\
\hline $\mathrm{p} 22(\mathrm{p} 9, \mathrm{p} 12)^{c}$ & \multicolumn{2}{|c|}{-} & - & - & 13.944 \\
\hline
\end{tabular}

Note, ${ }^{a}$ periods in hour. ${ }^{b}$ The $\mathrm{n}^{\text {th }}$ order harmonic of period $\mathrm{pl}$ is denoted by $\mathrm{n}^{\text {th }} \mathrm{pl} .{ }^{c} \mathrm{ID} \mathrm{pl}(\mathrm{pm}, \mathrm{pn})$ means the beat period between pm and pn based on the formula: $p l^{-1}=p m^{-1}-p n^{-1}$. The integers $1, \mathrm{~m}$ and $\mathrm{n}$ refer to the order ID. ${ }^{d}$ the thruster period. ${ }^{e}$ the sub-harmonic of the 6 -hr thruster period.

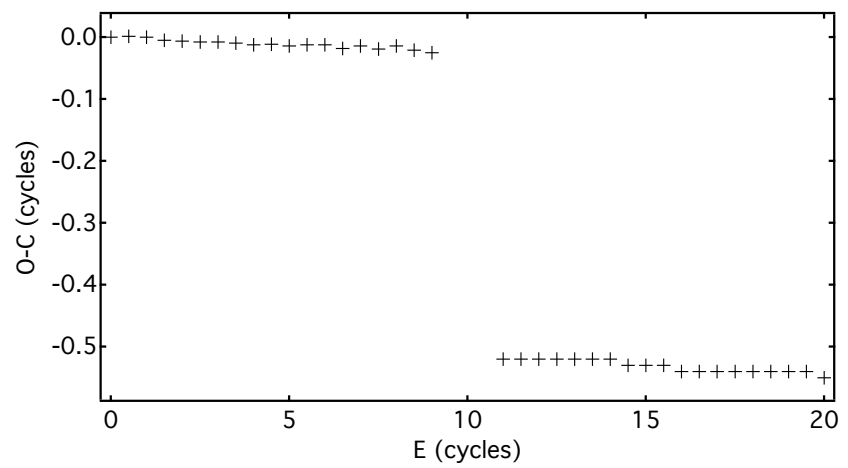

Figure 5: The O-C diagram for the 38 minimum times of the dips of TW Vir plotted in Figure 4. The time span of a cycle is 3.9256 day. 
Considering that the length of the normal outburst of UV Gem detected in K2-C0 was about 5 days and the time resolution of the K2 light curve observed in LC model was 29.4 min, a moving window for calculating a two-dimensional power spectrum should not be too small. The whole K2 light curve of UV Gem was separated into 8 equal parts. This means that the moving window is 4.7 day, which coincidently segregates the normal outburst from the quiescence. The light curve between the two dash lines shown in Figure 6 is the normal outburst part. A two-dimensional periodogram is shown in Figure 6. Obviously, the two significant and coherent modulations (i.e., double lines) at the periods of $2.07 \mathrm{hr}$ and $2.12 \mathrm{hr}$, which are the same as the results derived by Dai et al. (2016) [11], completely disappear during the normal outburst. Moreover, no periodic signals are seen in the power spectrum during outburst. This suggests that the orbital modulations of UV Gem in quiescence can completely disappear during a normal outburst. The hot spot on the accretion disk as a light source of the orbital modulations in a quiescent dwarf nova can be overwhelmed by the increased brightness of the entire accretion disk during the outburst. Hence, the lack of any orbital modulation in the periodogram during outburst further supports the thermal limit-cycle instability within the disk instability model [24].

Inspection of Figure 6 indicates that these two modulations before the outburst are stronger than after outburst. Before the normal outburst, the signal at the long period $2.12 \mathrm{hr}$ is gradually becoming stronger, while the relatively stronger signal at the short period $2.07 \mathrm{hr}$ almost remains constant. Although both modulations immediately reappear after the outburst, the signal at the long period $2.12 \mathrm{hr}$ repeats the previous variation cycle (i.e., it is becoming stronger and stronger like before outburst), while the signal at the short period $2.07 \mathrm{hr}$ switches into a much lower level than that before the outburst. Besides these two significant signals, there are the other two weaker but consistent signals at the periods of $1.06 \mathrm{hr}$ and $0.91 \mathrm{hr}$, which were not shown in the periodogram of the whole K2 light curve of UV Gem previously obtained by Dai et al. (2016) [11]. The former period $1.06 \mathrm{hr}$ is a first harmonic of the $2.12 \mathrm{hr}$ period. The latter period $0.91 \mathrm{hr}$ is close to the $5^{\text {th }}$ harmonic of the satellite thruster period (i.e., p2 listed in Table 2).

\section{Conclusion}

The four faint CV light curves observed in K2-C1 including three of our own deduced K2 light curves and a SFF corrected light curve of the dwarf nova J1125-0016 with a high improvement percentage $325.8 \%$ in photometric precision were used to search for potential periods, which may be due to jitters of the spacecraft. The periodogram of J1125-0016 may demonstrate a resonance phenomenon since the $88 \mathrm{~min}$ orbital period derived by Marsh et al. (2002) [20] is almost equal to one fourth of the 6-hr thruster period. Eight significant signals were picked out, of which 6 were doubleline peaks (two peaks refer to the orbital and thruster period and its harmonics, respectively). An accurate period of 3.9256 day can be derived from the 38 dips shown in the SC light curve of TW Vir, which was observed in $\mathrm{K} 2-\mathrm{C} 1$. Inspection of the two-dimensional periodogram of the dwarf nova UV Gem observed in K2-C0 shown in Figure 6 clearly indicates that two significant signals at the periods $2.07 \mathrm{hr}$ and $2.12 \mathrm{hr}$ only appear in quiescence and totally disappear in the normal outburst. The former one almost remains constant, while the latter one intensifies approaching the outburst. Moreover, the two weaker and shorter periods were first detected in this power spectrum. 


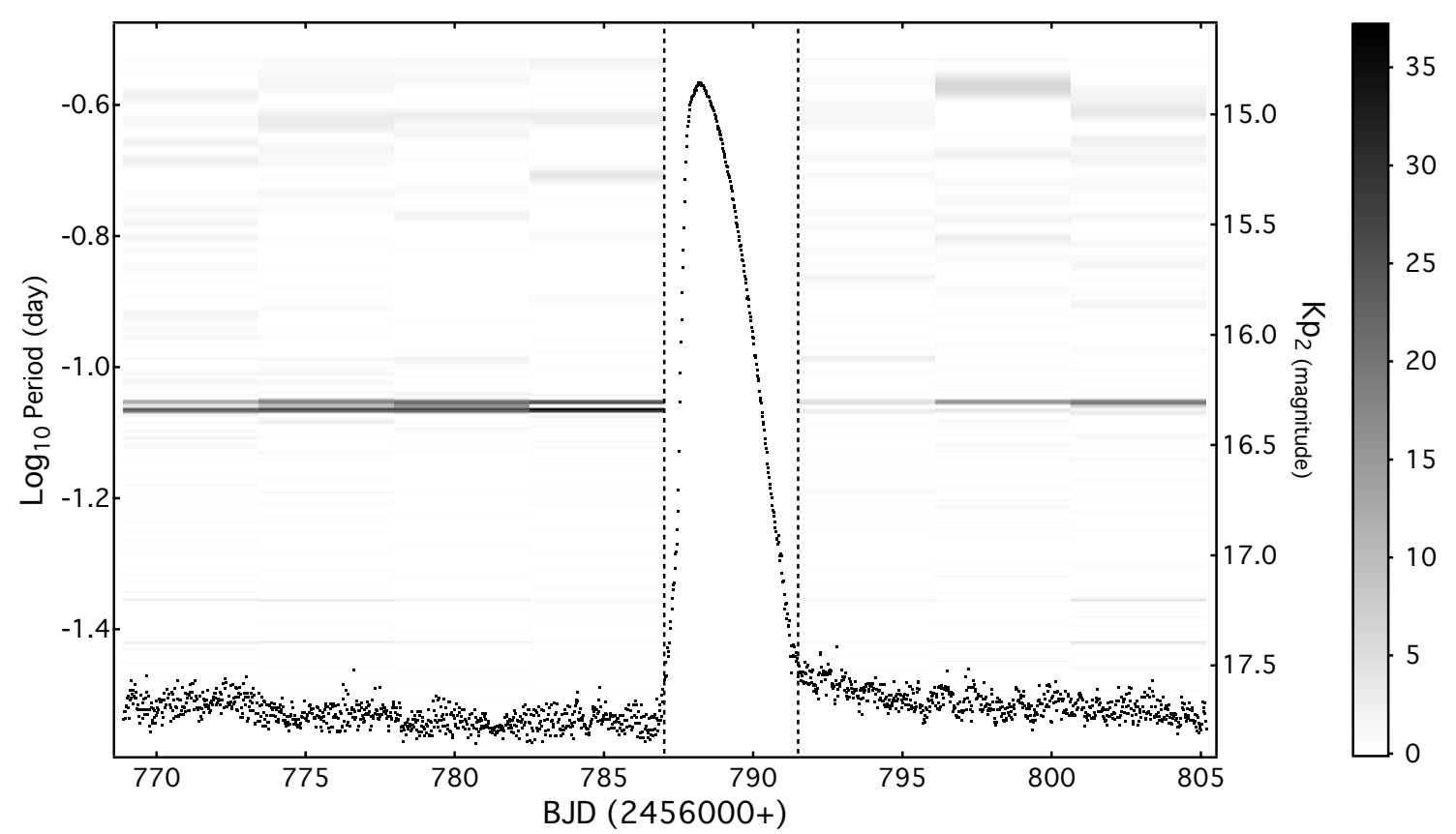

Figure 6: Two-dimensional power spectrum of dwarf nova UV Gem calculated from a moving window of 4.7 day is plotted with the K2 light curve in magnitude. The two dash lines segregate the outburst and quiescent parts of light curve.

The longer period $1.06 \mathrm{hr}$ was identified as the first harmonic of the 2.12 -hr period, and the shorter period $0.91 \mathrm{hr}$ may originate from the 5 th harmonic of the 6-hr thruster period.

\section{Acknowledgements}

Thanks Steve B. Howell for relating the cause of 4-day period shown in the SC data of K2-C1. The author gratefully acknowledges the support of K. C. Wong Education Foundation, Hong Kong. This work was partly supported by West Light Foundation and 1-year Visiting Scholar Program of The Chinese Academy of Sciences during a visit to Professor Paula Szkody in the University of Washington. PS acknowledges support from NSF grant AST-1514737. MK and PG acknowledge support from the Naughton Foundation and the UCC Strategic Research Fund.

\section{References}

[1] B. Warner, Cataclysmic Variables, 1995, Cambridge: Cambridge Univ. Press.

[2] B. Warner, Cataclysmic Variables, 2003, Cambridge: Cambridge Univ. Press.

[3] Y. Osaki, \& T. Kato, The Cause of the Superoutburst in SU UMa Stars is Finally Revealed by Kepler Light Curve of V1504 Cygni, PASJ, 2013, (65), 50.

[4] Y. Osaki, \& T. Kato, A further study of superoutbursts and superhumps in SU UMa stars by the Kepler light curves of V1504 Cygni and V344 Lyrae, PASJ, 2014 (65), 15.

[5] S. B. Howell, et al., Spectroscopy of New and Poorly Known Cataclysmic Variables in the Kepler Field, AJ, 2013 (145), 109. 
[6] S. Scaringi, et al., Spectroscopic identifications of blue-Ho-excess sources in the Kepler field of view, MNRAS, 2013, (428), 2207.

[7] S. Scaringi, S., P. J. Groot \& M. Still, Kepler observations of the eclipsing cataclysmic variable KIS J192748.53+444724.5, MNRAS, 2013, (435), L68.

[8] M. A. Wood, M. D. Still, S. B. Howell, J. K. Cannizzo \& A. P. Smale, V344 Lyrae: A Touchstone SU UMa Cataclysmic Variable in the Kepler Field, ApJ, 2011, (741), 105.

[9] J. Cannizzo, A. P. Smale \& M. A. Wood, The Kepler Light Curves of V1504 Cygni and V344 Lyrae: A Study of the Outburst Properties, ApJ, 2012, (747), 117.

[10] J. Smak, On the Periods of Negative Superhumps and the Nature of Superhumps, Acta Astron., 2013, (63), 109.

[11] Z. B. Dai, P. Szkody, P. M. Garnavich, M. Kennedy \& M. Templeton, Cataclysmic Variables observed during K2 Campaigns 0 and 1, AJ, 2016, (152), 5.

[12] A. Vanderburg \& J. A. Johnson, A Technique for Extracting Highly Precise Photometry for the Two-Wheeled Kepler Mission, PASP, 2014, (126), 948.

[13] A. Vanderburg, Reduced Light Curves from Campaign 0 of the K2 Mission, arXiv, 2014, [arXiv:1412.1827 [astro-ph. IM] ].

[14] M. Still \& T. Barclay, PyKE: Reduction and analysis of Kepler Simple Aperture Photometry data, Astrophysics Source Code Library, 2012, record ascl:1208.004.

[15] H. Ritter \& U. Kolb, Catalogue of cataclysmic binaries, low-mass X-ray binaries and related objects (Seventh edition), $A \& A, \mathbf{2 0 0 3}$, (404), 301 (update RKcat7.21,2013).

[16] A. J. Drake, et al., First Results from the Catalina Real-Time Transient Survey, ApJ, 2009, (696), 870.

[17] P. Szkody, et al., Cataclysmic Variables from Sloan Digital Sky Survey. V. The Fifth Year (2004), AJ, 2006, (131), 973.

[18] P. Hakala, G. Ramsay, B. Thomas \& P. Charles, K2 and MAXI observations of Sco X-1 evidence for disc precession?, MNRAS, 2015, (453), L6.

[19] A. L. Kraus, et al., The Mass-Radius Relation of Young Stars. I. USco 5, an M4.5 Eclipsing Binary in Upper Scorpius Observed by K2, ApJ, 2015, (807), 3.

[20] T. R. Marsh, et al., A first look at cataclysmic varible stars from the $2 d F$ QSO survey, ASPC, 2002, (261), 200.

[21] T. Kato \& U. Makoto, Outburst Characteristics of Three Likely SU UMa-type Dwarf Novae: UV Gem, FS And and AS Psc, IBVS, 2001, (5158), 1.

[22] T. Kato, T., et al., Survey of Period Variations of Superhumps in SU UMa-Type Dwarf Novae. IV. The Fourth Year (2011-2012), PASJ, 2013, (65), 23.

[23] B. Stolz \& R. Schoembs, The SU UMa star TU Mensae, A\&A, 1984, (132), 187.

[24] Y. Osaki, An accretion model for the outbursts of U Geminorum stars, PASJ, 1974, (26), 429.

[25] M. Otulakowska-Hypka, \& A. Olech, On supercycle lengths of active SU UMa stars, MNRAS, 2013, (433), 1338. 


\section{DISCUSSION}

JOSEPH PATTERSON: For the $2.1 \mathrm{hr}$ period in UV Gem, do you know when the previous superoutburst occurred?

ZHIBIN DAI: Until now, there is not enough data to derive the supercycle length [25]. The previous superoutburst was recorded in 2011 by Kato et al. (2013) [22]. After searching the AAVSO database of UV Gem, we found an outburst event around JD 2456670 recorded before $\mathrm{K} 2-\mathrm{C} 0$. But it is hard to judge it as a superoutburst or not since the too sparse observations. Hence, we concluded that there was not any strong evidence to support a superoutburst occurring before $\mathrm{K} 2-\mathrm{C} 0$. 